Internist 2021 · 62 (Suppl 1):S90-S96

https://doi.org/10.1007/s00108-021-00962-w

Angenommen: 21. Januar 2021

Online publiziert: 26. Februar 2021

(c) Springer Medizin Verlag $\mathrm{GmbH}$, ein Teil von

Springer Nature 2021

Redaktion

M. Raspe, Berlin

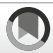

\section{8/w mit akutem und chronischem Husten}

\section{Vorbereitung auf die Facharztprüfung: Fall 16}

\author{
V. Rüttermann \\ Hausärztliche Gemeinschaftspraxis, Drensteinfurt-Walstedde, Deutschland
}

\section{Prüfungssimulation}

\section{Fallschilderung}

Eine 78-jährige Patientin ( $165 \mathrm{~cm}, 123 \mathrm{~kg}$, Body-Mass-Index $46 \mathrm{~kg} / \mathrm{m}^{2}$ ) stellt sich in der Hausarztpraxis mit seit 16 Wochen bestehendem trockenem Husten vor, der sich seit drei Tagen kontinuierlich verschlechtere.

Anamnese. Kein Kontakt zu Coronavirus-disease-2019(COVID-19)Patienten bekannt, kein Fieber, keine Gliederschmerzen, keine Rhinitis, keine Angina pectoris, Dyspnoe wird zunächst verneint. Eine kompensierte Herzinsuffizienz ist bekannt. Bei Voruntersuchungen normfrequenter Sinusrhythmus.

Vormedikation. Angiotensin-converting-enzyme(ACE)-Hemmer (Ramipril $10 \mathrm{mg}$ 1-0-0), Kalziumantagonist (Amlodipin $10 \mathrm{mg}$ 1-00 ), Diuretikum (Torasemid $5 \mathrm{mg}$ 1-0-0), Glucagon-like-peptide1-Agonist (Dulaglutid 1,5 mg 1-mal/Woche), Sodium-glucosecotransporter-2-Inhibitor (Empagliflozin 25 1-0-0), Metamizol ( $500 \mathrm{mg}$ bei Bedarf), unregelmäßig inhalative Kortikosteroide/lang wirksame $\beta 2$-Adrenergika (Fluticason $100 \mu \mathrm{g} /$ Vilanterol $39,82 \mu \mathrm{g}$, Hausstauballergie - allergisches Asthma bronchiale), bei Sodbrennen bedarfsweise Pantoprazol $20 \mathrm{mg}$.

Untersuchungsbefund. Blutdruck $120 / 84 \mathrm{mmHg}$, Pulsfrequenz $82 /$ min, absolut arrhythmisch, dezentes Giemen bds., Atemfrequenz $16 / \mathrm{min}$, Temperatur $36,8^{\circ} \mathrm{C}$, Sauerstoffsättigung $96 \%$, periphere Ödeme bds., Varikose, bei kurzem Hals und Adipositas permagna Jugularvenen eingeschränkt beurteilbar, erhebliche Gangstörung bei Z.n. Hüfttotalendoprothese und Adipositas - Angaben zu Belastungsdyspnoe daher nicht ausreichend verwertbar.

Laborbefunde. Rachenabstrich für "severe acute respiratory syndrome coronavirus 2" (SARS-CoV-2) negativ (Antigenschnelltest), C-reaktives Protein (CRP) $37 \mathrm{mg} / \mathrm{l}$ (Normbereich $<5 \mathrm{mg} / \mathrm{l}$ ), Blutkörperchensenkungsgeschwindigkeit (BSG) $60 \mathrm{~mm} / \mathrm{h}$ (Normbereich bei Frauen $>50 \mathrm{~J}$. $<30 \mathrm{~mm} / \mathrm{h}$ ), Leukozyten $4200 / \mu \mathrm{l}$ (Normbereich $4000-10.000 / \mu \mathrm{l}$ ), Prokalzitonin $0,08 \mathrm{ng} / \mathrm{ml}$ (Normbereich $<0,25 \mathrm{ng} / \mathrm{ml}$ ).
? Prüfungsfragen

- Welche Aspekte sollten bereits zu Beginn der Anamnese bedacht werden, wenn sich ein Patient mit dem Symptom Husten vorstellt?

- Ändert sich die Wahrscheinlichkeit für eine Ursache mit der Dauer der Symptomatik?

- An welche bedrohlichen Erkrankungen sollten Sie initial bei akutem Husten auch denken?

- Welche Labordiagnostik ist bei akutem Husten initial sinnvoll?

- Welche Rolle spielt die Lungenfunktionsprüfung bei akutem Husten?

- Ist die Aufzeichnung eines Elektrokardiogramms (EKG) bei akutem Husten sinnvoll?

- Wo befinden sich „Hustenrezeptoren“?

- Welche ätiologischen und diagnostischen Aspekte bedenken Sie bei subakutem Husten?

- Wie gehen Sie diagnostisch bei chronischem Husten vor?

- Welche Bedeutung hat ein gastroösophagealer Reflux als Ursache für Husten? 
? Welche Aspekte sollten bereits zu Beginn der Anamnese bedacht werden, wenn sich ein Patient mit dem Symptom Husten vorstellt?

- Gerade bei multimorbiden Patienten gilt: Akut Wichtiges muss von aktuell weniger Wichtigem differenziert werden, Leitsymptome - hier Husten - müssen definiert werden.

- Das Symptom „Husten“ unterliegt in hohem Maße einer individuell unterschiedlichen Wahrnehmung. Es ist daher unerlässlich, das Symptom unter Einbeziehung psychosozialer und soziokultureller Informationen individuell zu interpretieren. Eine Vielzahl völlig unterschiedlicher Erkrankungen kommt als mögliche Ursache infrage.

- Es gilt, die Vielzahl theoretisch möglicher Ursachen schnell auf wenige wahrscheinliche Differenzialdiagnosen zu reduzieren; für diese Ranking formulieren.

Dies wird erleichtert, wenn sich die Diagnostik an dem zu erwartenden ätiologischen Spektrum der Altersgruppe im entsprechenden Versorgungsbereich orientiert. Beispielsweise ist ein Infekt als Hustenursache bei jungen Patienten wahrscheinlicher als eine chronisch-obstruktive Lungenerkrankung (COPD).

\begin{tabular}{|c|c|c|}
\hline \multirow{5}{*}{$\begin{array}{l}\text { Akuter } \\
\text { Husten }\end{array}$} & \multirow{5}{*}{$\begin{array}{l}\text { Bis } \\
2 \text { Wo- } \\
\text { chen }\end{array}$} & (Virale) Erkältungsinfekte \\
\hline & & Allergische Rhinokonjunktivitis \\
\hline & & Asthma \\
\hline & & Aspiration: oft Kinder 1-3 Jahre \\
\hline & & Inhalative Intoxikation: Unfälle, Brände \\
\hline \multirow{6}{*}{$\begin{array}{l}\text { Subakuter } \\
\text { Husten }\end{array}$} & \multirow{6}{*}{$\begin{array}{l}\text { 2-8 Wo- } \\
\text { chen }\end{array}$} & Postvirale Rhinosinusitis \\
\hline & & $\begin{array}{l}\text { Postinfektiöser Husten mit vorübergehender } \\
\text { bronchialer Hyperreagibilität }\end{array}$ \\
\hline & & Pertussis, Adenoviren- oder Mykoplasmeninfekt \\
\hline & & Erkrankungen der Lunge/Pleura \\
\hline & & Pneumonie \\
\hline & & Pleuritis \\
\hline \multirow{13}{*}{$\begin{array}{l}\text { Chroni- } \\
\text { scher } \\
\text { Husten }\end{array}$} & \multirow[t]{13}{*}{$\begin{array}{l}>8 \text { Wo- } \\
\text { chen }\end{array}$} & $\begin{array}{l}\text { Chronische nichtobstruktive Bronchitis, chro- } \\
\text { nisch-obstruktive Lungenerkrankung }\end{array}$ \\
\hline & & $\begin{array}{l}\text { Asthma, nichtasthmatische eosinophile Bron- } \\
\text { chitis }\end{array}$ \\
\hline & & Lungentumoren \\
\hline & & Obstruktives Schlafapnoesyndrom \\
\hline & & Infektionen, z. B. Tuberkulose \\
\hline & & Diffuse Lungenparenchymerkrankung \\
\hline & & Systemerkrankung mit Lungenbeteiligung \\
\hline & & Bronchiektasen, Bronchomalazie \\
\hline & & Zystische Fibrose \\
\hline & & Gastroösophageale Refluxkrankheit \\
\hline & & $\begin{array}{l}\text { Medikamentös ausgelöster Husten: z. B. ACE- } \\
\text { Hemmer }\end{array}$ \\
\hline & & Kardiale Erkrankungen mit Lungenstauung \\
\hline & & Chronischer idiopathischer Husten \\
\hline
\end{tabular}

? Ändert sich die Wahrscheinlichkeit für eine Ursache mit der Dauer der Symptomatik?

- Das Ursachenspektrum verlagert sich im Zeitverlauf entscheidend.

- Daher hilft die Frage nach der Dauer des Hustens bei der Eingrenzung der wahrscheinlichen Ursachen (• Tab. 1).

\section{Der Fall.}

- Bei der Patientin besteht ein chronischer Husten (>8 Wochen) mit akuter Verschlechterung seit drei Tagen.

- Es stellt sich die Frage: Ist die Zunahme der Hustensymptomatik durch eine Verschlechterung der seit mehreren Wochen bestehenden Ursache bedingt oder liegt eine akut aufgetretene Zweiterkrankung vor?

Da ein akuter Husten mit Abstand am häufigsten Symptom eines Infekts der oberen Atemwege ist, liegt es nahe, zunächst diese Ursache in Betracht zu ziehen. Dann sollten entsprechend der epidemiologischen Situation bei jeder Diagnostik auch entsprechende Infektionsschutzmaßnahmen ergriffen werden.

In einem kleinen Teil der Fälle liegt kein harmloser Infekt, sondern eine potenziell lebensbedrohliche Erkrankung zugrunde (•Tab. 2).

? An welche bedrohlichen Erkrankungen sollten Sie initial bei akutem Husten auch denken?

- Anamnese und körperliche Untersuchung müssen zuerst klären, ob Alarmzeichen vorliegen ( $\mathbf{A b b}$. 1).

- Besteht ausgeprägte Luftnot, ist zunächst eine sofortige stationäre Einweisung zu organisieren.

- Bereits die Inspektion beim Betreten des Sprechzimmers (Mobilität, Atemfrequenz, Gesichtsausdruck, Hautkolorit) liefert intuitiv wichtige Anhaltspunkte für nächste Versorgungsschritte.

- Wichtigster Aspekt der körperlichen Untersuchung: pulmonale und kardiale Auskultation (Atemgeräusch vorhanden? Giemen? Feinblasige Rasselgeräusche? Knistern?) Während Sensitivität und Spezifität der Auskultation nicht selten überschätzt werden, wird die Bedeutung der Messung von Atemfrequenz und Sauerstoffsättigung oft unterschätzt.

- Obligat: Palpation der Pulsfrequenz, Messung des Blutdrucks, Abklärung peripherer Ödeme, Erfassung der Körpertemperatur; zum Teil sind diese Parameter Teil des CRB-65-Scores, mit

Tab. 2 Verdachtsdiagnosen, die ein sofortiges Handeln erfordern Akute Linksherzinsuffizienz, z. B. auf dem Boden einer kardialen Ischämie, Herzrhythmusstörungen

Pneumonie

Lungenembolie

Asthmaanfall, exazerbierte chronisch-obstruktive Lungenerkrankung mit respiratorischer Insuffizienz

Pneumothorax 


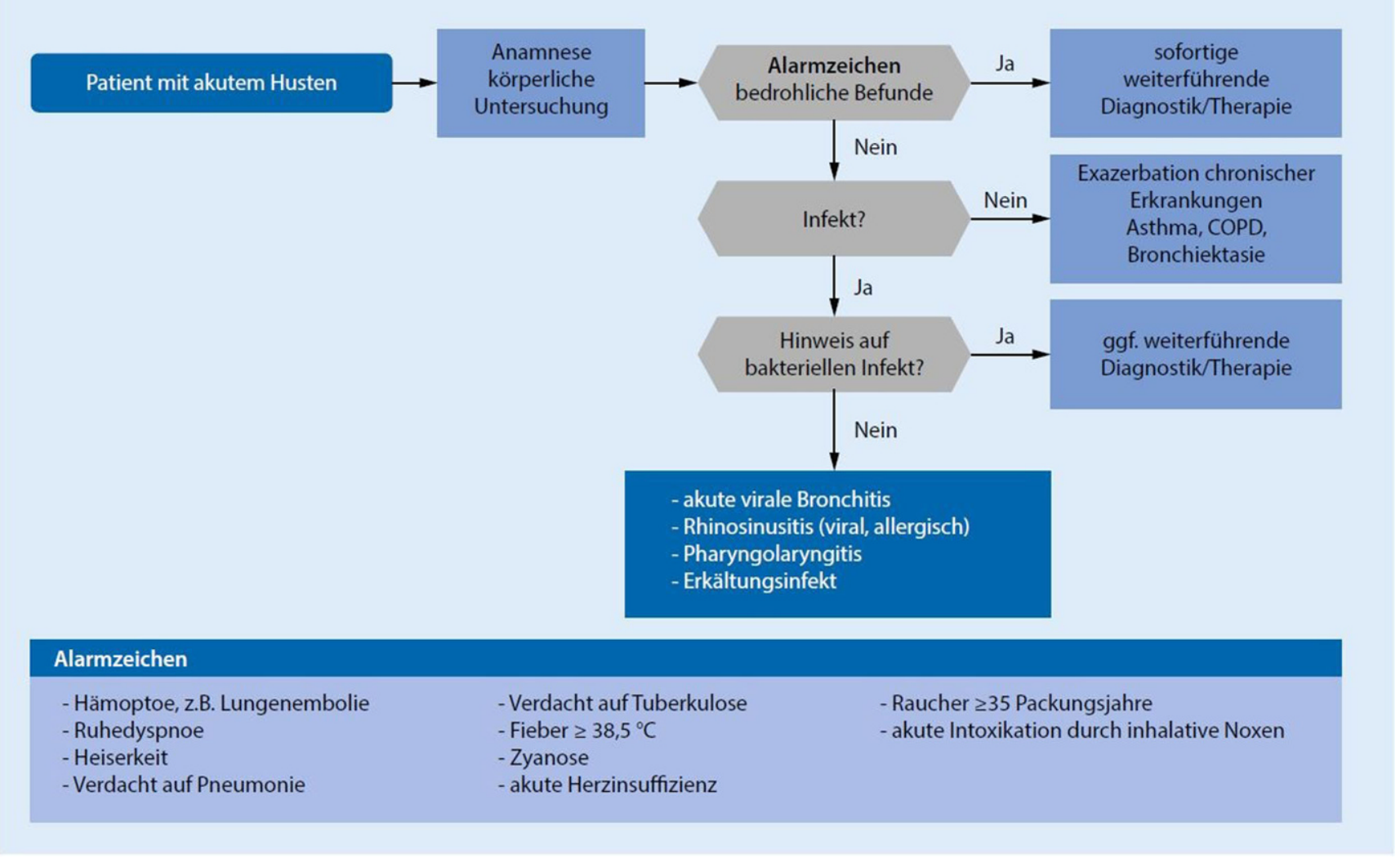

Abb. 1 \& Diagnostischer Algorithmus bei akutem Husten. COPD chronisch-obstruktive Lungenerkrankung. (Modifiziert nach

[3])

dessen Hilfe das Mortalitätsrisiko für Patienten mit Pneumonie abgeschätzt werden kann (CRB-65: „confusion, respiratory rate, blood pressure, age $\geq 65$ years"/Bewusstseinseinschränkung, Atemfrequenz, Blutdruck, Alter $\geq 65$ Jahre).

Merke. Nach der Beurteilung des möglichen Vorliegens von Alarmzeichen richtet sich im diagnostischen Algorithmus bei akutem Husten die Aufmerksamkeit auf die Abklärung eines Infekts (• Abb. 1).

\section{Cave.}

- Es kann zu kurzfristigen Verschlechterungen des Krankheitsverlaufs kommen; eine leichte und schwer erkennbare Lungenembolie kann z. B. Vorbote einer schweren Embolie sein $\rightarrow$ evtl. kurzfristige und aufmerksame Kontrollen.

- Patienten können eine eingeschränkte Wahrnehmung ihrer Körpertemperatur mit der Folge erstaunlicher Fehleinschätzungen haben $\rightarrow$ Temperaturmessung.

- Fieber kann insbesondere im hohen Alter z. B. bei Pneumonie fehlen.

- Giemen kann auch bei pulmonaler Stauung z. B. im Rahmen einer Linksherzinsuffizienz vorkommen und z. B. bei einem schweren Asthmaanfall fehlen.

- Rasselgeräusche können Ausdruck einer vorbestehenden Lungenerkrankung oder alter Veränderungen (Z. n. Pneumonie) sein, die nicht Ursache der aktuellen Dyspnoe sind.
- Pathologische Atemgeräusche sind nicht nur unspezifisch, sondern können z. B. bei Emphysem und geringer Inspirationstiefe abgeschwächt sein oder vollständig fehlen.

- Die Auskultation hat eine geringe "Eindringtiefe" von wenigen Zentimetern, erfasst also pathologische Prozesse, die in der Nähe der Thoraxwand liegen. Seltener wirken sich zentrale Veränderungen bis in die Peripherie aus (z. B. Verlegung eines Hauptbronchus, Fortleitung von Geräuschen) und verändern so das Atemgeräusch.

- Dyspnoe kann bei Adaptation des Patienten oder bei geringer Belastbarkeit fehlen, z. B. aufgrund orthopädischer Begleiterkrankungen wie im geschilderten Fall.

- Ein basal abgeschwächtes Atemgeräusch kann ebenso durch harmlosen Zwerchfellhochstand wie durch Atelektase oder Pleuraerguss bedingt sein.

- Geringgradige Pleuraergüsse, z. B. im Rahmen einer Lungenembolie, Pneumonie oder Tumorerkrankung, sind auskultatorisch und perkutorisch nicht erfassbar.

? Welche Labordiagnostik ist bei akutem Husten initial sinnvoll?

- Abhängig von der jeweiligen epidemiologischen Situation, der Verfügbarkeit von Testmöglichkeiten und der geltenden Testkriterien kann die möglichst frühzeitige Kontrolle auf SARS-CoV-2, z. B. mithilfe von Antigenschnelltests, erforderlich sein. 
Tab. 3 Risikokonstellationen, die besondere Aufmerksamkeit erfordern

Immunsuppression

Tumorerkrankung

Raucherstatus (>30 Packungsjahre)

Neurologische Erkrankung mit erhöhter Aspirationsgefahr

Herzinsuffizienz

Multimorbidität

Tuberkulosekontakt

- Bei akutem Husten ohne Alarmzeichen (z. B. ohne Atemnot, Hämoptoe, Thoraxschmerz, hohem Fieber, Hinweis auf Pneumonie): auf weitere technische Untersuchungen verzichten [3, 6]

- Ursache akuter Husten: häufig unkomplizierte virale Erkrankung (etwa 90\%) der oberen (Rhinosinusitis, Postnasaldrip-Syndrom, Pharyngitis) und/oder der unteren Atemwege (Tracheitis, Bronchitis)

- Keine Differenzierung zwischen viraler und bakterieller Genese möglich anhand des Kriteriums trocken/produktiv [3] oder aufgrund der Sputumfarbe und -konsistenz. Bei bakteriellen Infekten steigt das C-reaktive Protein zwar tendenziell stärker an als bei viralen Infekten. Dennoch ist dieser Parameter zur Differenzierung zwischen bakterieller und viraler Infektion nicht geeignet [3].

\section{Der Fall.}

- BSG und CRP erhöht

- Prokalzitonin und Leukozyten im Normbereich

- Verdacht auf viralen Infekt

- An die Kontrolle der pathologischen Werte sollte im Verlauf gedacht werden, um weitere Differenzialdiagnosen nicht zu übersehen.

- In diesem Fall: CRP normalisierte sich später vollständig.

- Die BSG fiel auf ein bei Adipositas Grad III seit vielen Jahren konstant gering erhöhtes Niveau.

Merke. Husten und Dyspnoe können Symptom einer Lungenembolie sein, einer der Erkrankungen, die am häufigsten übersehen werden. Besteht eine geringe bis mittlere Wahrscheinlichkeit, kann ein D-Dimer-Test zum Ausschluss einer Lungenembolie durchgeführt werden [1].

\section{? Welche Rolle spielt die Lungenfunktionsprüfung bei akutem Husten?}

Nach viralen Infekten gehören infektexazerbierte COPD und Asthma zu den häufigsten Ursachen für akuten Husten.

Der Fall. Bei der Patientin ist bei anamnestisch hyperreagiblem Bronchialsystem und vorbestehender intermittierender antiobstruktiver Therapie das bei Adipositas nur leise Giemen zunächst mutmaßlich auf eine erneute bronchiale Obstruktion zurückzuführen. Tatsächlich führt die Applikation von Salbutamol zu einer sofortigen partiellen Besserung. Erst jetzt bemerkt die Patientin eine Erleichterung der Atmung. In der Zusammenschau mit den am Folgetag vorliegenden Laborwerten kann die Hustensymptomatik zumindest partiell als Äquivalent einer Bronchokonstriktion bei Infektexazerbation interpretiert werden.

\section{Lungenfunktionsprüfung.}

- Bietet der enge Zeitrahmen der initialen Abklärung nach Ausschluss von COVID-19 die Möglichkeit, eine Lungenfunktionsprüfung durchzuführen, ist diese hilfreich.

- Pragmatisches Vorgehen bei Akutdiagnostik (z. B. Hausbesuch) und hoher Wahrscheinlichkeit für eine Bronchokonstriktion: Eine Broncholyse kann zunächst ohne Lungenfunktionsdiagnostik sinnvoll sein.

- Bei Anhaltspunkten, dass keine Erkältung oder bronchiale Obstruktion Ursache für die Hustensymptomatik ist, sind zeitnah weitergehende Untersuchungen erforderlich.

\section{? Ist die Aufzeichnung eines Elektrokardiogramms bei} akutem Husten sinnvoll?

Merke. Eine pulmonale Stauung kann zu Husten führen. Zur Erfassung möglicher Ursachen einer Herzinsuffizienz ist u.a. ein EKG erforderlich.

\section{Der Fall.}

- Bei der Patientin zeigt sich ein neu aufgetretenes Vorhofflimmern. Ein Hinweis auf kardiale Ischämie findet sich nicht. Das am Folgetag vorliegende stark erhöhte N-terminale pronatriuretische Peptid vom B-Typ (NT-proBNP; 5811 pg/ml; normal bis $612 \mathrm{ng} / \mathrm{ml}$ ) kann Ausdruck einer Herzinsuffizienz als Teilursache des Hustens sein. Eine Intensivierung der Herzinsuffizienztherapie (vorübergehende Erhöhung der Torasemiddosis von $5 \mathrm{mg}$ 1-0-0 auf $10 \mathrm{mg}$ 1-1-0, Umstellung von Ramipril $10 \mathrm{mg}$ auf Valsartan $320 \mathrm{mg}$ 1-0-0 und in den Folgetagen unter engmaschiger Blutdruckkontrolle schrittweise Umstellung auf Valsartan 102,8 mg/Sacubitril 97,2 mg bis 1-0-1) führt innerhalb von 10 Tagen zu einem vollständigen Sistieren der Hustensymptomatik, einer Besserung der peripheren Ödeme und einer Gewichtsreduktion von $4 \mathrm{~kg}$.

Cave. Vorhofflimmern führt ebenso wie eine chronische Nierenerkrankung - auch bei erhaltener linksventrikulärer Funktion - zu einer Erhöhung von NT-proBNP.

Merke. Oft kann nur eine Verdachtsdiagnose gestellt werden. Von besonderer Bedeutung sind daher angepasste Verlaufskontrollen, v. a. bei Vorliegen einer Risikokonstellation (- Tab. 3).

\section{? Wo befinden sich "Hustenrezeptoren"?}

- Die Stimulation von Hustenrezeptoren führt zur Auslösung des Hustenreflexes.

- Verantwortliche Nervenenden des N. vagus im Epithel der Atemwege, am Perikard und im Ösophagus. Sie werden durch mechanische, chemische und entzündliche Faktoren gereizt. Variabel sind Intensität des Triggers, Verteilung und Dichte der Rezeptoren, Schwelle für Reizauslösung am Rezeptor sowie periphere und zentrale Modulation der Nervenimpulse. 


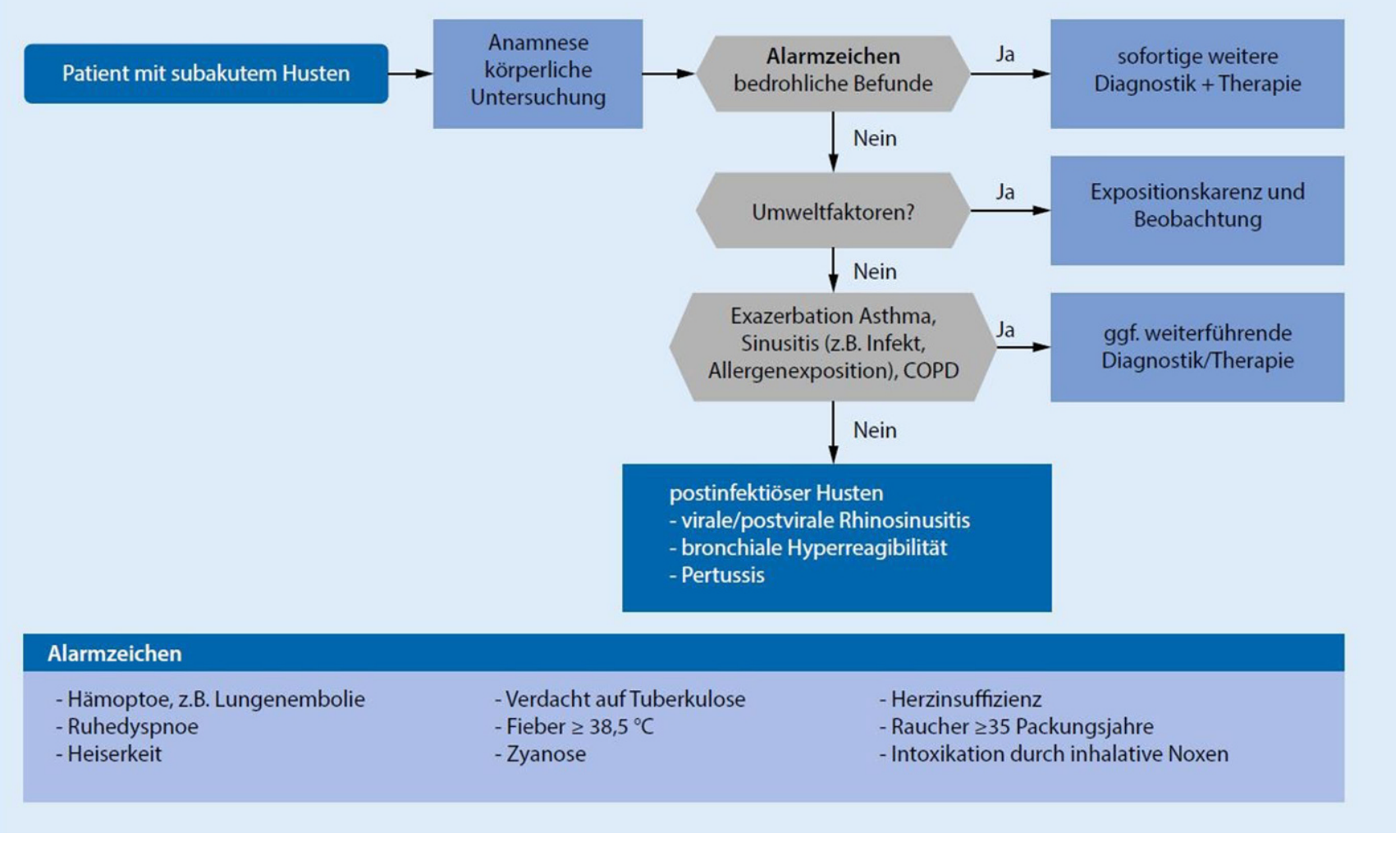

Abb. $2 \Delta$ Diagnostischer Algorithmus bei subakutem Husten. COPD chronisch-obstruktive Lungenerkrankung. (Modifiziert nach [3])

- Dies erklärt, warum auch eine Reihe extrapulmonaler Erkrankungen und psychischer Faktoren in die Differenzialdiagnostik einbezogen werden sollte.

- Ein „Landkartenalgorithmus" hilft, die häufig auf Bronchien verkürzte diagnostische Fokussierung zu erweitern und die Diagnostik an Verteilung und Dichte der Hustenrezeptoren zu orientieren.

? Welche ätiologischen und diagnostischen Aspekte bedenken Sie bei subakutem Husten?

- Die Hustensymptomatik kann abhängig vom Ausmaß der Epithelschädigung 6-12 Wochen andauern (v.a. Infektion durch Adenoviren, Mykoplasmen, Bordetella pertussis).

- Auch bei subakutem Husten ist zunächst auf mögliche Alarmzeichen zu achten ( $\bullet \mathbf{A b b}$. 2). Bei Fehlen einer Risikokonstellation ist eine weitergehende Diagnostik oft verzichtbar [3].

- Im Vergleich zum akuten Husten liegt bei prolongiertem Husten häufiger eine bronchiale Hyperreagibilität, postvirale Rhinosinusitis oder postinfektiös vermehrte Reizbarkeit der Hustenrezeptoren vor.

- An eine bisher möglicherweise nicht diagnostizierte COPD sollte wegen möglicher medikamentös-therapeutischer Konsequenzen und der eventuell intensivierten Empfehlung zum Nikotinverzicht gedacht werden.

- Gegebenenfalls ist eine Lungenfunktionsprüfung zur Abklärung restriktiver und obstruktiver Ventilationsstörungen sinnvoll.
- Bei Verdacht auf bronchiale Hyperreagibilität kann auch der Erfolg eines vorübergehenden Einsatzes inhalativer Kortikosteroide oder inhalativer $\beta 2$-Adrenergika bzw. bei Rhinosinusitis eines nasalen Kortikosteroids diagnostisch genutzt werden ([3]; - Abb. 2 und 3). Ggf. ist ein inhalativer Metacholinprovokationstest erforderlich. Eine Verlaufskontrolle nach 4-6 Wochen sollte klären, ob eine weitergehende Diagnostik erforderlich ist.

? vor?

- ACE-Hemmer senken auch bei Patienten, bei denen zuvor kein ACE-Hemmer-Husten bestand, die Schwelle für den Hustenreflex.

- Angiotensin-Il-Rezeptor-Subtyp-1-Blocker gehen mit einem geringeren Hustenrisiko einher als ACE-Hemmer ( $2 \%$ gegenüber bis zu $10 \%$ der Patienten), sodass bei Husten unter ACEHemmer eine Umstellung auf ein Sartan vor Durchführung einer weiteren Diagnostik erwogen werden sollte [3].

- Husten kommt u.a. auch als Nebenwirkung einer Therapie mit Betablockern, Amiodaron, Methotrexat, Gliptinen oder inhalativen Kortikosteroiden vor.

- Bei Husten länger als 8 Wochen: Einleitung einer Stufendiagnostik - zunächst mit Röntgenuntersuchung des Thorax und Lungenfunktionsprüfung

- Die Sensitivität der Röntgenuntersuchung des Thorax ist für Prozesse unter einer Größe von $2 \mathrm{~cm}$ gering. 


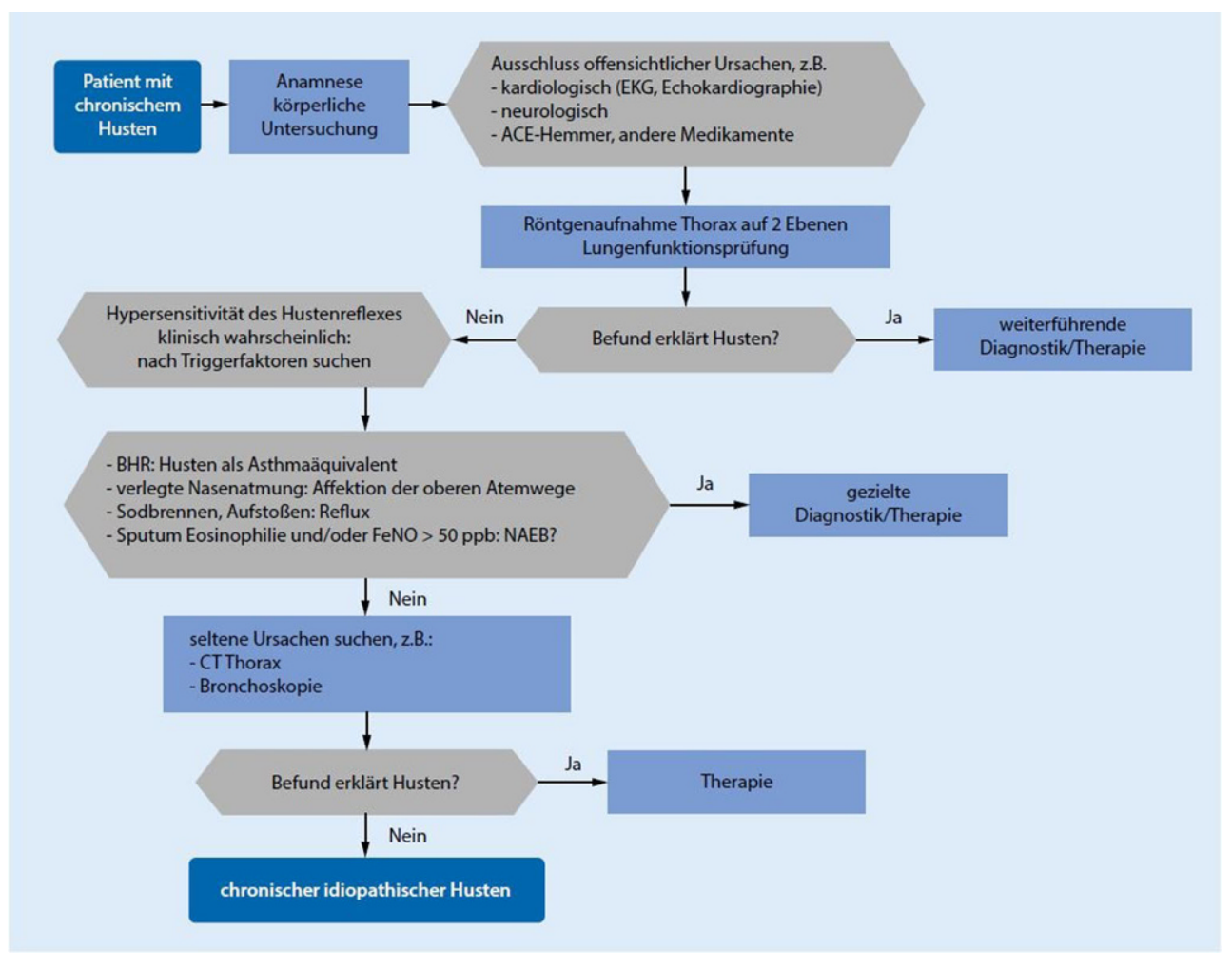

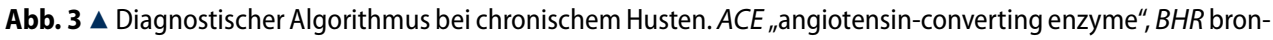
chiale Hyperreagibilität, CTComputertomographie, EKG Elektrokardiogramm, FeNOFraktion des exhalierten Stickstoffoxids, NAEB nichtasthmatische eosinophile Bronchitis. (Modifiziert nach [3])

- Eine Computertomographie (CT) des Thorax ist z. B. für die Detektion eines Bronchialkarzinoms - bei Männern die häufigste tumorbedingte Todesursache [7] - sehr viel sensitiver.

- Zu bedenken ist das nicht geringe Risiko einer Überdiagnostik mit entsprechenden Nachteilen; z. B. Pneumothorax bei Punktion eines in der Screening-CT gefundenen Rundherds, der sich bei der weiteren Abklärung in $>98 \%$ der Fälle als nichtmaligner Genese charakterisieren lässt [4].

- Bei hohem Risiko für Tumorerkrankung (z. B. Alter 55-74 Jahre, Nikotinkonsum > 30 Packungsjahre): positive Nutzen-RisikoBewertung bereits für Durchführung einer Screeninguntersuchung mittels Low-dose-CT $[2,5]$. Dies gilt umso mehr für die Abklärung bei symptomatischen Hochrisikopatienten.

\section{? Welche Bedeutung hat ein gastroösophagealer Reflux als Ursache für Husten?}

- Bei unauffälliger Lungenfunktionsanalyse und normalem Thoraxröntgenbefund: Reflux ist häufiger Trigger für Hustenreiz

- Selbst ein schwacher ösophagealer Reflux kann durch Reizung von Hustenrezeptoren im Ösophagus ein Trigger für chronischen Husten sein.

- Im geschilderten Fall: Husten sistierte vollständig ohne Intensivierung der Therapie mit Protonenpumpeninhibitor.

Cave. Fehlt eine typische Refluxsymptomatik wie Sodbrennen, erschwert dies die Diagnosestellung.
Schlüsselwörter. Respiratorische Zeichen und Symptome · Risikofaktoren · Bronchiale Hyperreagibilität · Herzinsuffizienz · Lungenfunktionsprüfung

\section{Korrespondenzadresse}

Dr. med. V. Rüttermann

Hausärztliche Gemeinschaftspraxis

Nordholter Weg 10, 48317 Drensteinfurt-Walstedde, Deutschland

ruettermann@gemeinschaftspraxis-walstedde.de

\section{Einhaltung ethischer Richtlinien}

Interessenkonflikt. V. Rüttermann gibt an, dass kein Interessenkonflikt besteht.

Für diesen Beitrag wurden vom Autor keine Studien an Menschen oder Tieren durchgeführt. Für die aufgeführten Studien gelten die jeweils dort angegebenen ethischen Richtlinien. Für Bildmaterial oder anderweitige Angaben innerhalb des Manuskripts, über die Patienten zu identifizieren sind, liegt von ihnen und/oder ihren gesetzlichen Vertretern eine schriftliche Einwilligung vor.

The supplement containing this article is not sponsored by industry.

\section{Literatur}

1. AWMF (2015) Diagnostik und Therapie der Venenthrombose und der Lungenembolie. S2k-Leitlinie. https://www.awmf.org/leitlinien/detail/ll/065-002. html.Zugegriffen:21. Nov. 2020

2. IQWiG (2020) Lungenkrebsscreening mittels Niedrigdosis-Computertomografie. Institut für Qualität und Wirtschaftlichkeit im Gesundheitswesen. https:// www.iqwig.de/download/s19-02_lungenkrebsscreening-mittels-low-dose-ct_ abschlussbericht_v1-0.pdf?rev=144033.Zugegriffen: 13. Feb. 2021 


\section{Facharzt-Training}

3. Kardos P, Dinh QT, Fuchs K-H, Gillissen A, Klimek L, Koehler M, Sitter H, Worth H (2019) Leitlinie der Deutschen Gesellschaft für Pneumologie und Beatmungsmedizin zur Diagnostik und Therapie von erwachsenen Patienten mit Husten. Pneumologie 73(3):143-180. https://doi.org/10.1055/a-0808-7409

4. Kauczor H-Uea (2015) Lungenkrebsscreening: Wann und für wen? Dtsch Aerztebl. https://doi.org/10.3238/PersPneumo.2015.10.02.02

5. Leitlinienprogramm Onkologie der AWMF, Deutsche Krebsgesellschaft, Deutsche Krebshilfe S3-Leitlinie Prävention, Diagnostik, Therapie und Nachsorge des Lungenkarzinoms: Langversion 1.0; AWMF-Registernummer 020/007OL. https:// www.leitlinienprogramm-onkologie.de/fileadmin/user_upload/Downloads/ Leitlinien/Lungenkarzinom/LL_Lungenkarzinom_Langversion_1.0.pdf. Zugegriffen: 13. Feb.2021

6. Morice AH, Fontana GA, Belvisi MG, Birring SS, Chung KF, Dicpinigaitis PV, Kastelik JA, McGarvey LP, Smith JA, Tatar M, Widdicombe J (2007) ERS guidelines on the assessment of cough. Eur Respir J 29(6):1256-1276. https://doi.org/10.1183/ 09031936.00101006

7. RKI Krebs in Deutschland für 2015/2016. Eine gemeinsame Veröffentlichung des Robert Koch-Instituts und der Gesellschaft der Epidemiologischen Krebsregister in Deutschland e.V. Robert Koch-Institut, Gesellschaft der Epidemiologischen Krebsregister in Deutschland. https://www.krebsdaten.de/Krebs/DE/Content/ Publikationen/Krebs_in_Deutschland/kid_2019/krebs_in_deutschland_2019. pdf?_blob=publicationFile

Hier steht eine Anzeige.

Springer 